\title{
Théodore Monod (1902-2000) et l'archéologie bretonne : note sur un épisode méconnu de la vie du « fou du désert »
}

Théodore Monod (1902-2000) and Breton archaeology: notes on a little known episode of the life of "the madman of the desert"

Theodore Monod (1902-2000) y la arqueología de Bretaña: nota sobre un episodio poco conocido de la vida del "loco del desierto"

Marie-Yvane Daire, Elías López-Romero et Catherine Le Gall

\section{OpenEdition}

Journals

Édition électronique

URL : http://journals.openedition.org/rao/2193

DOI : 10.4000/rao.2193

ISBN : 978-2-7535-3432-2

ISSN : 1775-3732

Éditeur

Presses universitaires de Rennes

\section{Édition imprimée}

Date de publication : 25 décembre 2013

Pagination : 289-301

ISBN : 978-2-7535-3430-8

ISSN : 0767-709X

Référence électronique

Marie-Yvane Daire, Elías López-Romero et Catherine Le Gall, «Théodore Monod (1902-2000) et l'archéologie bretonne : note sur un épisode méconnu de la vie du « fou du désert » », Revue archéologique de l'Ouest [En ligne], 30 | 2013, mis en ligne le 25 décembre 2015, consulté le 05 décembre 2020. URL : http://journals.openedition.org/rao/2193; DOI : https://doi.org/10.4000/rao. 2193

Ce document a été généré automatiquement le 5 décembre 2020.

Tous droits réservés 


\title{
Théodore Monod (1902-2000) et l'archéologie bretonne : note sur un épisode méconnu de la vie du «fou du désert »
}

\author{
Théodore Monod (1902-2000) and Breton archaeology: notes on a little known \\ episode of the life of "the madman of the desert" \\ Theodore Monod (1902-2000) y la arqueología de Bretaña: nota sobre un episodio \\ poco conocido de la vida del "loco del desierto"
}

Marie-Yvane Daire, Elías López-Romero et Catherine Le Gall

Si la vie de ce savant et naturaliste français a marqué le $\mathrm{xx}^{\mathrm{e}}$ siècle, notamment par ses nombreux travaux effectués dans le Sahara, on connaît moins l'épisode au cours duquel Théodore Monod a côtoyé quelques-uns des pionniers de l'archéologie bretonne: ce sont eux qui, à l'aube des années 1920, entraînèrent le jeune homme sur plusieurs chantiers de fouille de la région où il exerça ses talents de fouilleur, d'observateur, de naturaliste, d'anthropologue, de dessinateur et aussi, accessoirement, de poète. De cette expérience "d'archéologue breton ", demeurée si discrète dans une biographie foisonnante, il gardera pourtant un goût définitif pour la préhistoire.

Dès le mois d'avril 1918, Théodore Monod s'engage dans la rédaction de carnets qu'il tiendra jusqu'en 1934. En 1997, son fils, Cyrille Monod, en publie des extraits regroupés sous le titre: Les carnets de Théodore Monod. Dans l'intimité du "fou du désert " (Monod, 1997). L'article que nous proposons aujourd'hui a débuté par l'exploitation des quelques notes, récits, poèmes et dessins regroupés dans ce livre que nous avons analysés et qu'il nous a très vite fallu recouper avec d'autres sources: des données historiographiques régionales (projet ICARE), ceci afin d'éclairer les récits du jeune chercheur, d'identifier les personnages, les sites visités... Piquée au vif, notre curiosité nous a ensuite entrânés sur les traces de Th. Monod, jusque dans ses archives privées les plus intimes (journaux et correspondances conservés au Muséum national d'histoire naturelle), à la recherche 
des indices qui nous permettent aujourd'hui de livrer ce chapitre inédit de la vie d'un infatigable voyageur.

\section{Une brève « tranche » de la vie de Théodore Monod}

On ne saurait évoquer cet épisode breton de la vie de Th. Monod sans rappeler le parcours et les grandes étapes de l'existence de cet «éternel étudiant » (Berne et Monod [dir.], 2010). Né en 1902 à Rouen, Th. Monod est le troisième fils de Wilfred Monod, pasteur et théologien protestant dont l'influence spirituelle et culturelle a joué un rôle fondamental dans l'élaboration de sa personnalité (fig. 1). Si, après l'obtention de son baccalauréat, à l'âge de 16 ans, il hésite un temps entre la théologie et les sciences naturelles, c'est vers ces dernières qu'il se tourne finalement. Il leur consacrera sa vie.

Figure 1 : Portrait de Théodore Monod avant son embarquement pour la mission océanographique sur l'Andrée III (1922).

Figure 1 : Portrait of Théodore Monod, before boarding on Andrée III ship for the oceanographic expedition (1922).

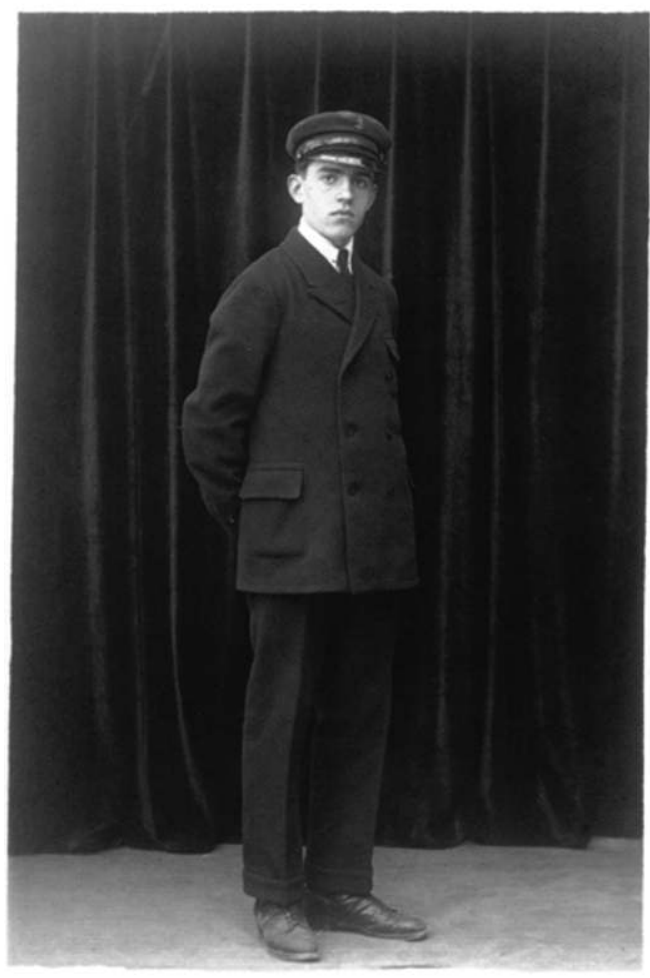

Archives privées, avec l'aimable autorisation de A. Monod.

4 En 1920, Th. Monod passe ses certificats de licence de zoologie et de botanique; il est alors choisi par le ministère de la Marine pour effectuer une première croisière océanographique en 1920, qui sera suivie de deux autres en 1921 et 1922. L'épisode «breton» de sa formation de biologiste marin, d'océanographe, mais aussi d'archéologue, se situe au cours de ces trois années ; nous y reviendrons plus en détail. Nommé ensuite assistant au Muséum d'histoire naturelle de Paris en 1922, il part pour sa première mission outre-mer, en Mauritanie, et traverse son premier désert. 
Il soutient sa thèse en 1926 (Monod, 1926), puis il participe, en tant que naturaliste, à la mission d'exploration Augiéras-Draper qui va parcourir durant quinze mois le Sahara occidental. Sa vocation de naturaliste trouve là un terrain d'élection: le zoologiste devient géologue, botaniste, archéologue, préhistorien... et le Sahara ne le quittera plus. Dès lors, des dizaines de voyages se succéderont.

6 En 1938, Th. Monod est affecté à Dakar pour créer et diriger l'Institut français d'Afrique noire (IFAN) où sont étudiées toutes les disciplines des sciences naturelles et des sciences humaines, et qui rassemble des collections de plantes, d'animaux, de matériel ethnologique et archéologique ; il y restera jusqu'en 1965.

7 Th. Monod poursuit parallèlement ses recherches sur la faune marine : il est nommé, en 1942, directeur du laboratoire des pêches d'outre-mer au Muséum, participe aux essais du premier bathyscaphe (1954), puis est nommé membre de l'Académie des sciences en 1965. S'ensuivront des expéditions en Iran, à Madagascar, aux Açores, au Yémen du Sud, dans le désert de Lybie et en Mauritanie où il cherche inlassablement la météorite de Chinguetti. Son voyage terrestre s'achève en 2000.

8 Pour revenir plus en détail sur l'épisode de la vie de Th. Monod qui nous intéresse ici, celui des années 1920-1922, ses biographes n'ont généralement retenu que l'aspect biologique et océanographique de ses recherches (Berne et Monod [dir.], 2010 ; Cans, 2002 ; Collectif, 2010; Hureau et Escudier, 2005 ; Jarry, 2001 ; Leca et Jacquard, 1997 ; Vray, 1994). Ce sont donc tout d'abord les extraits de ses carnets, publiés par son fils Cyrille (Monod, 1997), que nous avons tenté de recouper avec plusieurs publications archéologiques, dues notamment à Charles Bénard (Bénard et al., 1921, 1922 ; Bénard, 1929) et à l'abbé Favret (Favret et al., 1923). Quelques difficultés rencontrées dans la reconstitution des faits, des lieux, des dates, nous ont conduits à remonter à la source de l'information et aux archives personnelles de Th. Monod, déposées depuis 2003 avec le fonds scientifique au Muséum d'histoire naturelle à Paris. Si depuis sa plus tendre jeunesse Th. Monod tenait des « carnets » aussi intimes que scientifiques, ils prendront, au cours de ses expéditions, la forme particulière de "correspondances». Dans la période qui nous intéresse, il s'agit de (longues) lettres presque quotidiennes qu'il adresse à ses parents : «Bien Aimés, aurez-vous la bonté de me garder les pages que je vous envoie? Je les réunirai plus tard en journal »(Roscoff, lettre du 14 juillet 1920).

Ce sont donc ces diverses sources que nous avons recoupées pour démêler le déroulement des faits de cet épisode breton très bref et pourtant d'une extraordinaire richesse scientifique mais aussi historiographique.

En effet, ces documents relatent une série de rencontres, d'où naîtront quelques " portraits » qui intéressent très directement l'histoire de la recherche régionale et des pionniers de l'archéologie nationale (Daire et López-Romero, 2011 ; López-Romero et Daire, à paraître). En apportant un éclairage sur l'implication personnelle de Th. Monod dans des recherches sur divers sites archéologiques du sud du département du Finistère, ces sources témoignent aussi de la découverte d'une nouvelle discipline, par le jeune chercheur, et des enthousiasmes intellectuels qu'elle n'a pas manqué de susciter. 


\section{Océanographie et archéologie : des rencontres décisives}

11 Année 1920 : pour la première fois depuis la guerre, la Société océanographique de France (SOF) prépare une nouvelle campagne de recherche et arme des bateaux. Dans le port de Saint-Servan (Ille-et-Vilaine), tandis que le trois-mâts barque Pourquoi pas? prépare son armement sous l'autorité du commandant Charcot, le contre-torpilleur Mistral termine le chargement des appareils scientifiques de pêche et de sondage en vue d'une mission océanographique de trois mois dans l'Atlantique.

Pendant ce temps, Th. Monod, étudiant à la Sorbonne, prépare sa licence-ès-sciences naturelles. Même si la veille de l'examen du certificat de zoologie, il se fait conduire au poste de police du Panthéon pour «avoir déchiré une affiche pornographique » en revenant de l'observatoire, cela ne l'empêche pas d'être reçu premier « avec mention très bien et 32 points d'avance» (W. Monod, "Journal d'enfance» de Th. Monod, dimanche 20 juin 1920).

13 Suite à ses brillants résultats au certificat de zoologie - mais aussi à celui de botanique obtenu le 6 juillet - ses maîtres sont si contents de lui qu'il est désigné officiellement comme « naturaliste » pour la croisière océanographique à bord du Mistral, de mi-juillet à fin septembre 1920. Son père précise fièrement qu'il a même été préféré à un autre candidat, agrégé de sciences, âgé de 28 ans et élève de l'École normale.

Arrivé le 13 juillet à la station de biologie marine de Roscoff (Finistère), il y passera d'abord quinze jours pour se préparer à ses nouvelles fonctions sur le Mistral.

Au lendemain d'une guerre qui a sinistré, entre autres corps de métiers, celui des marins pêcheurs bretons, le but de la mission océanographique du Mistral est d'étudier l'amélioration des engins de pêche, ainsi que " la destruction de certains cétacés ennemis de la sardine et pourfendeurs de filets » (Maratray, 1920a et 1920b) tout particulièrement dans la région des Glénan: trois cents stations et quinze cents relevés d'algues seront réalisés au cours de cette campagne : "Je "fais" du plankton deux fois par jour » écrira-t-il à ses parents le 8 août 1920 , alimentant ainsi le futur mémoire de recherche pour lequel il est missionné (Monod, 1921 et 1922).

Th. Monod profite de son séjour à Roscoff, dans l'attente de son embarquement, pour étudier la faune marine du nord Finistère. "À propos de bateau, Bénard m'annonce qu'il viendra me chercher à domicile avec son torpilleur : c'est une agréable économie de temps et d'argent » (Th. Monod, Roscoff, 18 juillet 1920).

Cette note fait référence à un épisode décisif: c'est en effet à cette époque que Th. Monod va côtoyer de plus près le commandant Charles Bénard, président de la Société d'océanographie de France (SOF), qu'il a probablement rencontré quelque temps auparavant à Paris et le marquis de la Réveillère, vice-président, embarqués à bord du navire de la Marine nationale Mistral, commandé par le lieutenant de vaisseau Masse. Il fera également la connaissance de l'abbé Favret, aumônier du bord avec qui il aura des échanges théologiques nourris.

Si C. Bénard et J. de la Réveillère sont des officiers de marine réputés, ils sont aussi des pionniers des investigations archéologiques dans l'Ouest de la France et seront, avec d'autres, à l'origine de la création du Groupe finistérien d'études préhistoriques (GFEP) 
en 1923 puis du musée préhistorique finistérien de Saint-Guénolé Penmarc'h (cf. infra ; López-Romero et Daire, 2013).

Pour en revenir au contexte de la rencontre et à la mission océanographique, le Mistral quitte la rade de Brest le 19 juillet avec, à son bord, trois civils chargés de la mission scientifique: M.M. Maratray, Hamel et Monod. Mais le navire est peu adapté à la navigation, proche des côtes, notamment dans le secteur des Glénan; l'équipage doit alors s'organiser et s'adapter. Au mois d'août 1920, l'organisation est la suivante: "Mardi, mercredi, jeudi, vendredi: travail aux Glénans [sic], mais coucher à terre, à Concarneau. Samedi, dimanche, lundi à Saint-Guénolé. [...] J'habite à l'hôtel à Saint-Guénolé où j'ai ici un laboratoire épatant. » (Th. Monod, à bord du Mistral, 8 août 1920).

Penmarc'h-Kérity sera en effet la base terrestre d'installation du laboratoire de la mission océanographique, ce qui éclaire l'importance des recherches archéologiques menées à cette époque dans la région de La Torche (cf. infra)! Du 21 au 30 août 1920, Monod et le reste de l'équipe séjourneront ainsi à Penmarc'h, avant de se rendre à Lorient pour la « quinzaine du poisson », d'où ils rayonneront et visiteront Belle-île-enMer, l'île de Groix...

21 À l'origine de l'expérience archéologique bretonne de Th. Monod, il y a donc sa rencontre décisive avec quelques pionniers de la préhistoire finistérienne et, notamment, leur chef de file, Ch. Bénard (dit Bénard Le Pontois, 1867-1931) (fig. 2), officier de marine mais aussi passionné de Préhistoire. Ce dernier lui présente plusieurs de ses confrères, officiers de marine, mais aussi ses amis archéologues, dont notamment l'abbé Favret (1875-1950) (fig. 3), membre du GFEP (Groupe finistérien d'études préhistoriques) qui sera également le fondateur scientifique du musée d'Épernay suite aux nombreuses fouilles qu'il a conduites sur les nécropoles de la Marne. 
Figure 2 : Portrait de Ch. Bénard Le Pontois par R. Cocheril en 1927. Figure 2 : Portrait of Ch. Bénard Le Pontois by R. Cocheril in 1927.

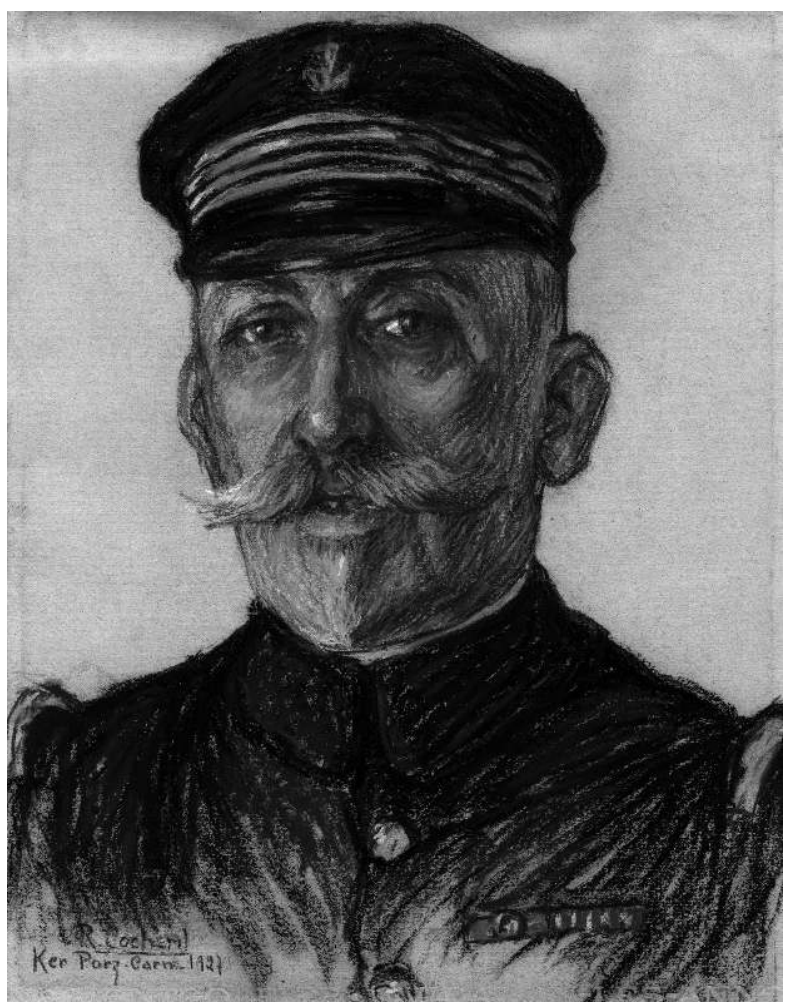

(c) Labo Archéosciences UMR 6566 CReAAH. 
Figure 3 : Excursion archéologique. Années 1920. Premier personnage à droite : l'abbé Favret. Figure 3 : Archaeological excursion. 1920's. First person on the right: Abbé Favret.

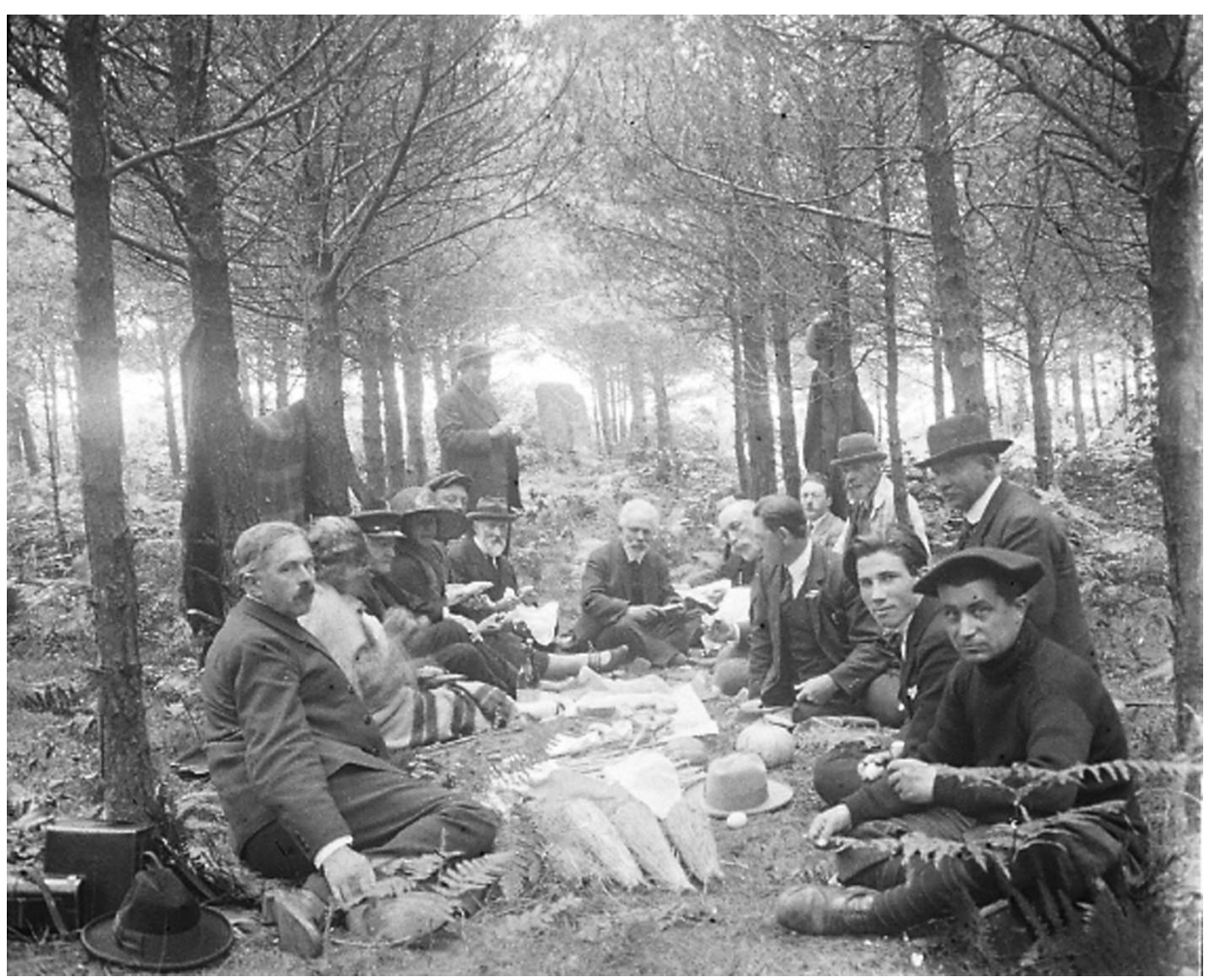

Coll. Ch. Bénard (c) Labo Archéosciences UMR 6566 CReAAH.

Relatant l'une de ses premières sorties "archéologiques» en compagnie des personnages mentionnés précédemment, Th. Monod note : «Nous étions 3: Bénard, un abbé [il s'agit vraisemblablement de Favret, NDR] et moi et vous auriez ri de voir le papiste, l'athée et le parpaillot s'extasier en chœur devant un silex bien ouvragé » (20 juillet 1920). Ils feront plus ample connaissance dans les jours qui suivent et Th. Monod dressera de ses compagnons des portraits sans complaisance : «Au carré du Mistral, nous nous trouvons : l'abbé Favret, sec, fertile en anecdotes, ne dédaignant pas l'alcool; le commandant Masse, petit homme rouge, le sceptique personnifié; l'administrateur à trois galons Andrieux, sorte de fou nationaliste se plaisant à revenir sur les horreurs de la guerre; l'administrateur à deux galons Broucorsse, mêmes idées plus estompées, être plus indécis, souvent de l'avis du dernier intervenant; le commandant Besnard Le Pontois, homme propre, anti-tsariste à tout crin, antialcoolique, scientifique et intelligent mais jurant comme un païen. Voilà le cadre ou plutôt le jeu de quilles dans lequel je suis tombé, et vous pensez leur étonnement à la vue d'un jeune homme ne buvant pas, ne fumant pas, ne disant pas de cochonneries, etc. » (25 juillet 1920).

Son opinion est peu amène lorsqu'il s'agit de l'amiral de la Réveillère (fig. 4), fantasque préhistorien qui proposa de ré-empiler les parties du grand menhir brisé de Locmariaquer à Paris, face à l'obélisque de Louxor, place de la Concorde, ou devant la tour Eiffel, pour l'Exposition universelle de 1900 (Réveillère, 1903). S'ensuit une levée de boucliers régionaux contre ce projet qui aurait dépossédé le golfe d'un de ses trésors (Chaigneau et al., 2009, p. 442 et 453). Le témoignage du jeune ascète qu'est Th. Monod est sans appel : " Je vois à Lorient beaucoup de gens et je découvre l'humanité plus laide que je ne la connaissais: des goinfres et des débauchés [...]. Il y a ici un marquis de La Réveillère, épouvantable goinfre et fin diseur de cochonneries qui a été chargé de me faire oublier des 
principes qu'on me trouve trop absolus. Mais je ne me laisse pas faire et ils n'arriveront pas à me faire boire la moindre fine ou à dire la moindre obscénité. Ce marquis est gastronome, il en est répugnant : il a une cave de 10000 bouteilles, certaines de 1865, c'est révoltant. Et je commence à comprendre la nécessité d'une révolution. Rencontré ici un médecin qui me prêche la débauche et ne peut concevoir que l'on soit encore propre de corps et d'âme à 18 ans. Le reste à l'avenant... » (Lorient, 30 août 1920). Ses relations avec l'abbé Favret seront cordiales et riches de discussions théologiques : "Je suis presque "chrétien" au dire mon aumônier l'abbé Favret, avec lequel j'ai eu cet après-midi un tournoi oratoire pas banal... » (20 août 1920). La personnalité la plus marquante du groupe est sans conteste Ch. Bénard avec qui Th. Monod aura les relations les plus suivies au cours de la saison 1920.

Figure 4 : Portrait de l'amiral J. de la Réveillère. Figure 4 : Portrait of the amiral J. de la Réveillère.

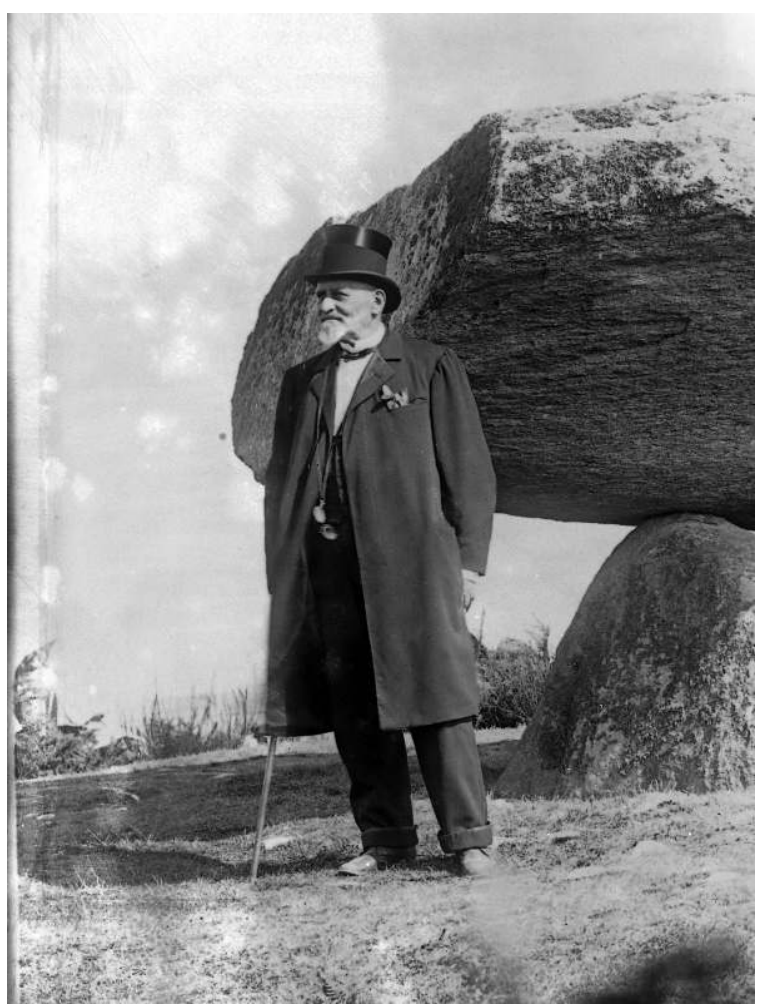

Coll. Ch. Bénard (C) Labo Archéosciences UMR 6566 CReAAH.

Ch. Bénard, né à Redon le 14 février 1867, passe son baccalauréat à Bordeaux et entre ensuite à l'École navale (1883). À Bordeaux, il occupe une série de postes en rapport avec l'administration maritime et, en 1897, fonde la Société océanographique du golfe de Gascogne ; cette société sera transformée en 1921 en Société océanographique de France. Il publie toute une série de livres et d'articles sur ce sujet ainsi que sur la région de Bordeaux. Il effectue une série de missions dans l'océan glacial arctique, dont la première, en 1908, à bord du Jacques Cartier. Cette expédition fut organisée par la Société océanographique sous le patronage de S.A.S. le Prince de Monaco qui, en ces débuts $\mathrm{du} \mathrm{xx}$ siècle, jouera un rôle très important dans le développement des sciences en France. À partir de 1919, et après la mort de Louis Le Pontois (commandant et archéologue actif dans la région de Lorient dès 1894), il adopte le nom BénardLe Pontois qu'il utilise cependant uniquement dans le milieu de l'archéologie. Ch. Bénard fait partie du noyau de chercheurs du Groupe finistérien d'études 
préhistoriques (1923). Très rapidement, le GFEP bénéficiera d'une reconnaissance nationale et se constituera en Institut finistérien d'études préhistoriques dès 1926 (Desailly, 1926). En 1929 est publié Le Finistère préhistorique, un livre qui résulte de la collaboration de plusieurs membres du groupe mais que Bénard signe finalement comme seul auteur (Bénard, 1929). Le livre inclut une préface de Louis Capitan, célèbre docteur et préhistorien décédé en 1929, à qui Bénard succédera comme professeur à l'école d'anthropologie de Paris.

Dans ses "carnets", c'est bien sur le personnage de Ch. Bénard que Th. Monod s'attarde le plus, en lui témoignant une certaine admiration, notamment pour son côté "globe-trotter» qu'il lui envie déjà : "Bénard, au point de vue des voyages, est très intéressant : il connaît Terre-Neuve ; les îles aléoutiennes, le Labrador, la Nouvelle-Zemble [!], la Russie, l'Algérie, la Tripolitaine, le Sénégal, l'Afrique orientale, le Brésil, la Colombie britannique, etc., etc. Il est spécialiste en pays arctiques et dans quelques années, il repartira pour longer la côte de Sibérie, la Russie au Kamtchatka. Il veut tout spécialement étudier la région du globe où le glaciaire n'a pas fondu!»(21 août 1920). Il consacre ainsi plusieurs pages de son manuscrit à rapporter, dans les lettres à ses parents, les récits et aventures de Ch. Bénard en Russie notamment.

D'un point de vue plus personnel, Th. Monod note, au début de leur rencontre, que

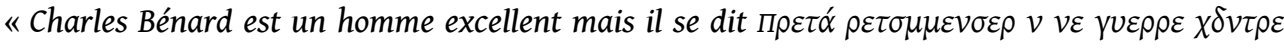

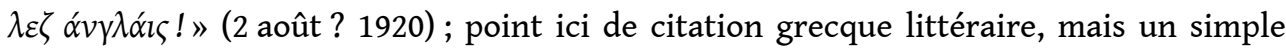
message " codé » dont la transcription est " prêt à recommencer une nouvelle guerre contre les Anglais ».

27 Mais au-delà du voyageur, c'est le préhistorien Bénard qui fascine le jeune Monod : « Le Cdt Bénard s'occupe activement de préhistoire en son royaume et il a trouvé moult crânes, silex, dolmens et autres vieilleries. [...] Je vous ai dit qu'au point de vue de la préhistoire, ce pays est plus que merveilleux. Bénard qui excelle dans cette science me raconte beaucoup de choses : il a découvert une foule de mégalithes, des crânes, des squelettes, des enceintes néolithiques, des pierres à cupules, des pierres à sacrifice [!], etc. " (8 août 1920). Certains passages témoignent encore de cette admiration respectueuse: "Je vous envoie un article de Bénard qui est intéressant en tant que donnant une vue générale sur cette extraordinaire région de la presqu'île de Penmarc'h. Il y a des passages bien écrits. " (29 septembre 1920). Il s'agit probablement de l'article paru dans le Bulletin de la société archéologique du Finistère en 1920, qui relatait la campagne de 1919 (Bénard et al., 1920).

28 À travers les récits des expériences de terrain, nous verrons infra l'importance qu'a eu cette rencontre dans la vie intellectuelle et scientifique du jeune naturaliste, conscient aussi du rôle alors tenu par son mentor: "De retour à Paris, le Cdt Bénard veut me faire faire la connaissance de S. Reinach et de M. Boule, l'anthropologiste. Je crois que si [le mot est souligné quatre fois par Monod] je me mettais hardiment à la remorque de Bénard, j'aurais les relations que je voudrais dans le monde de la pensée et de la science, et par là, bien des portes ouvertes » (vendredi matin, [s. d., probablement en septembre 1920], à bord du Mistral). L'histoire nous raconte que Th. Monod suivra finalement une autre voie... 


\section{Le « pied à l'étrier »: Théodore Monod, archéologue en Bretagne}

29

Finistère, principalement la région de Roscoff et l'île de Batz, les missions du Mistral les conduiront dans le sud Finistère (Penmarc'h, Concarneau, l'archipel des Glénan) et jusque dans le Morbihan. Cette découverte de la région va se doubler de l'initiation de Th. Monod à la discipline archéologique, puisqu'il va participer à des fouilles et prospections en pays bigouden.

\section{Les débuts... à la pointe de La Torche}

$\mathrm{Au}$ titre des difficultés rencontrées dans la restitution des événements proposée ici figure le manque de précision dans la chronologie des recherches de terrain relatées par Ch. Bénard, conjuguées à quelques confusions possibles dans certains courriers de Th. Monod à ses parents, lettres qui ont généralement été postdatées ; un autre écueil réside dans le fait que les lettres de Th. Monod, pourtant prolixes et descriptives, ne mentionnent pratiquement jamais le nom des sites décrits; et c'est donc par recoupements de dates et de descriptions de trouvailles que nous en proposons une attribution. Ainsi, nous proposons d'identifier le lieu de découverte décrit par Th. Monod lors de l'une des premières expéditions comme étant celui de la pointe de La Torche, mais dans ce cas, la chronologie de la lettre, datée du 8 août, doit en fait remonter au 18 ou 19 juillet (cf. supra) : "Il [Bénard] va m'emmener demain visiter en qualité de savant un Kjokennmoeding, c'est-à-dire les détritus de cuisine de nos vénérables ancêtres néolithiques. Je serai en mesure après cette visite de vous dresser le menu de ces intéressants précurseurs. Je vais tâcher de m'emparer de quelques pièces et je suis sûr que papa sera ému par la palpation de ces coquillages dévorés par les Néolithiques. Combien j'aimerais vous montrer ce que je vois... » (Lettre datée du 8 août mais probablement antérieure).

L'expérience de Th. Monod en matière de préhistoire bretonne débutera donc par une visite approfondie de la presqu'île de La Torche, à Penmarc'h en juillet 1920. Cette visite sera pour Ch. Bénard l'occasion de compléter son inventaire de l'année précédente (carte mégalithique de la région de La Torche dressée par le Cdt Bénard en 1919, in Bénard et al., 1920). Cette carte révisée sera publiée en 1921 sous l'appellation «Carte mégalithique de la presqu'île de Penmarc'h, dressée par le Cdt Bénard et Th. Monod », in Bénard et al., 1921, planche 1).

En effet, la première mention d'une intervention archéologique de terrain, dans les lettres de Th. Monod à ses parents, remonte au 20 juillet 1920, c'est-à-dire qu'elle suit immédiatement son départ de Roscoff à bord du Mistral : « Hier après-midi, nous avons été à la presqu'île de La Torche, endroit mystérieux où les civilisations se sont succédé, depuis le silex préhistorique jusqu'au corps de garde de Louis XV, en passant par le poignard en (----) [mot illisible], le mégalithe et la poterie gallo-romaine. Nous avons travaillé à la pioche le Kjokennmoeding où l'on trouve des vertèbres de poissons, des os, des mollusques variés servant de nourriture aux préhistoriques. Je vous en rapporterai des échantillons. [...] Il y a aussi sur cette presqu'île un reste d'allée couverte et un atelier de taille de silex vraiment prodigieux par le nombre de pièces qu'on y rencontre. On trouve aussi des morceaux de poteries néolithiques et si on ouvrait la butte, il y a certainement des chambres funéraires à [déc]ouvrir. Quelle région passionnante et comme j'aimerais vous montrer ces merveilles. » (20 juillet 1920).

Revue archéologique de l'Ouest, 30 | 2013 

passage, pour le curieux menhir "anthropo ou plutôt gyno morphique» de Kervédal à Penmarc'h (Bénard et al., 1921, p. 25), autour duquel une fouille rapide sera menée. « Ce matin, nous avons fouillé sous le fameux menhir anthropomorphique. La présence d'un lit de galets surmontant une argile stérile en débris d'industrie humaine me fait sérieusement croire que le dit menhir n'a jamais été dressé. Tout autour, (mais pas dessous) on recueille à foison des ossements d'animaux, les poteries néolithiques (type gris et type rouge), les poteries galloromaines, moyenâgeuses à émail, enfin modernes.» (Dimanche, 13 heures, seconde quinzaine de septembre.) Ces recherches ne manquent pas d'alimenter la ferveur du fils de pasteur qu'est Th. Monod pour qui «ces fouilles sont vraiment bien passionnantes et religieuses» (8 septembre 1920) et lui inspirent de longues pages de réflexions théologiques et philosophiques.

\section{Théodore Monod : de Roz an Trémen...}

Comme en attestent la publication de 1921 et les relevés cosignés par Th. Monod (Bénard et al., 1921), ce dernier participe très activement à la campagne de fouilles de l'été 1920 sur le cimetière de l'âge du Fer de Roz an Trémen à Plomeur (fig. 5, fig. 6 et fig. 7$)$; cette nécropole fut la première à révéler le rôle funéraire des stèles en pierre retrouvées en abondance dans l'ouest de la péninsule (Daire, 2005). En effet, la présence d'au-moins deux stèles cannelées sur le site, ajoutée au nombre important d'embases signalées par les fouilleurs, a permis à Ch. Bénard de démontrer l'association entre stèles et nécropoles en Armorique à l'époque gauloise (Bénard, 1929). La fouille (1919-1920) et les sondages qui suivirent $(1925,1926$ et 1927) consistèrent à creuser autour des stèles ou des embases où des regroupements d'urnes et de dépôts osseux furent observés ; ainsi, autour de la grande stèle cannelée, découverte fortuitement, la fouille révéla une série de quatre incinérations en urnes, trois poches d'ossements incinérés déposés directement en terre, un crâne isolé et une inhumation. Dès lors, l'existence d'une ou plusieurs stèles devint un indicateur archéologique susceptible de révéler l'existence d'un site à vocation funéraire. Quatre stèles, sur les six mentionnées par les fouilleurs, sont encore conservées actuellement: trois d'entre elles au Musée préhistorique finistérien et une au Musée départemental breton de Quimper. Lors de la découverte de la première stèle en 1919, les archéologues ont recueilli les propos d'un agriculteur local qui leur certifiait «que deux autres pierres semblables, placées en triangle avec la première, avaient été détruites à la dynamite » (Bénard, 1929, p. 186-187). 
Figure 5 : Plan de localisation de la nécropole de Roz an Trémen à Plomeur (Finistère) par Th. Monod.

Figure 5 : Location map of the Roz an Trémen cemetery, Plomeur (Finistère) by Th. Monod.

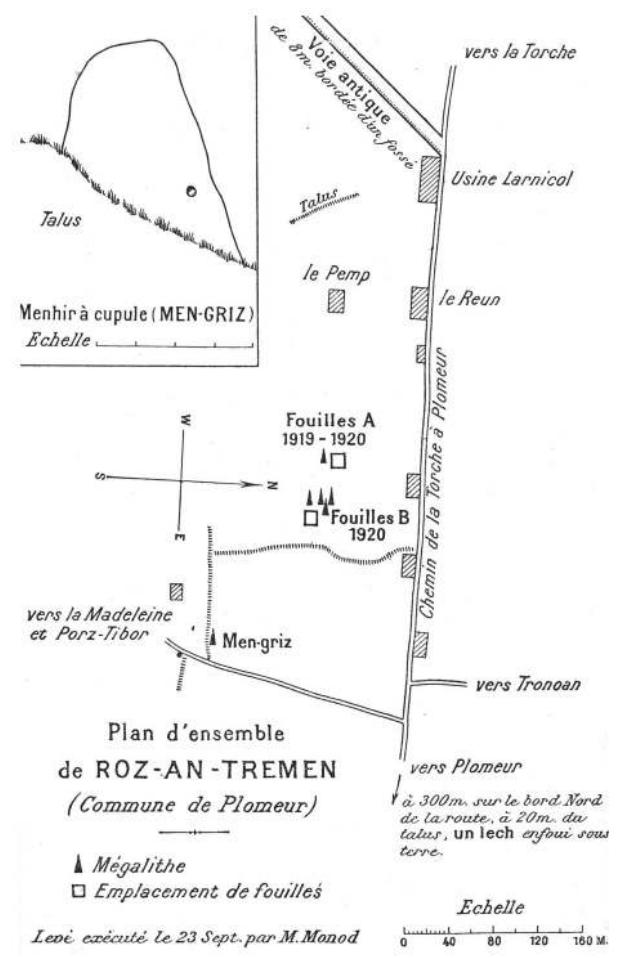

In Bénard et al., 1921, pl. 4 
Figure 6 : Plan d'ensemble de la nécropole de l'âge du Fer de Roz an Trémen à Plomeur (Finistère) par Th. Monod.

Figure 6 : General map of the Roz an Tremen Iron Age cemetery, Plomeur (Finistère) by Th. Monod.

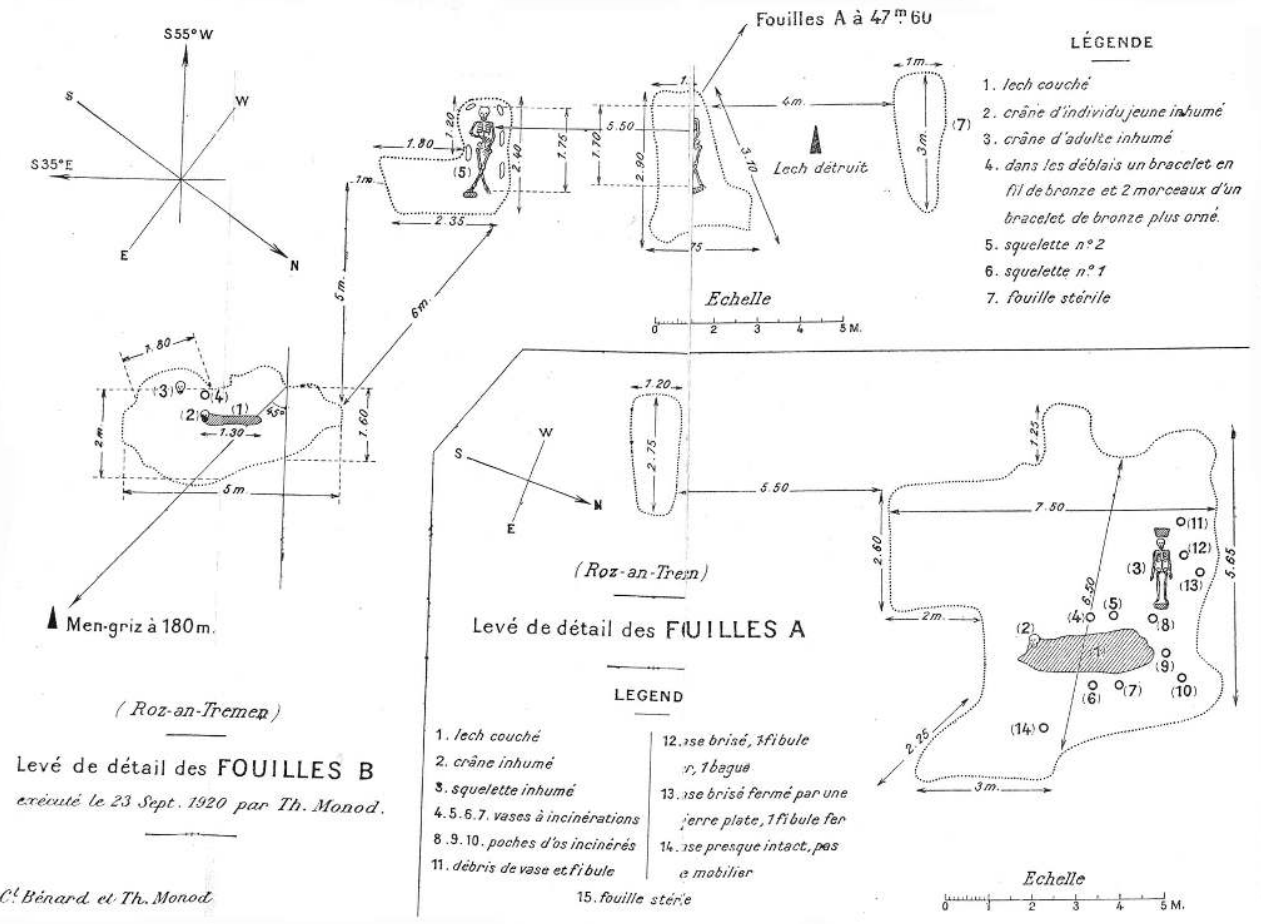

In Bénard et al., 1921, pl. 5. 
Figure 7 : Dessins de crânes humains provenant de la nécropole médiévale de Saint-Urnel (en haut) et de celle l'âge du Fer de Roz an Trémen (en bas), à Plomeur (Finistère), par Th. Monod. Figure 7 : Drawings of skulls from the medieval Saint-Urnel cemetery and Iron Age Roz an Trémen cemetery, Plomeur (Finistère) by Th. Monod.

Planche 2

Crâne du squelette de la carrière de STURNEL

portant une perforation traumatique de la suture fronto-pariétalo

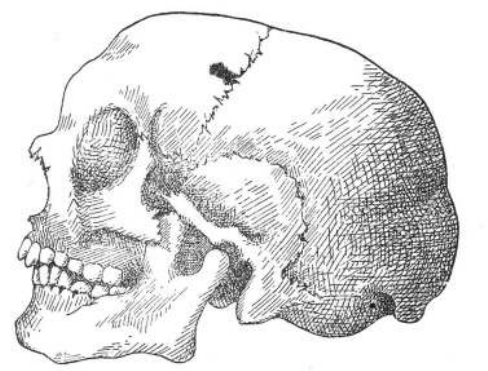

Cràne du squelette No:2 de ROZ - AN-TREMEN

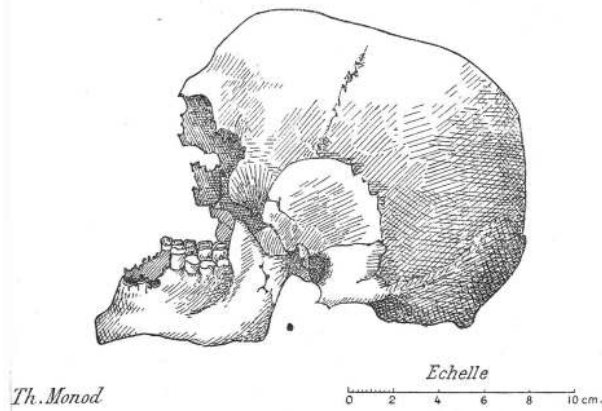

In Bénard et al., 1921, pl. 2.

C'est précisément un monument de ce type qui interpella le jeune Th. Monod qui participait aux fouilles. Voici les lignes qu'il consigna alors dans ses courriers : «Ce matin, j'ai participé à des fouilles mégalithiques qui ont mis au jour un menhir cannelé très ancien et un menhir phallique magnifique. Cette région est incroyablement riche : à chaque pas, on rencontre des fragments de poteries néolithiques, d'os, de résidus de cuisine. Hier après-midi, nous avons fait une tournée archéologique et nous avons vu la troublante silhouette du menhir anthropomorphique découvert par Besnard: ce colosse a six mètres de long, il est couché par terre. Cette presqu'île est un vaste cimetière et je suis plein d'une religieuse émotion à la vue de ces témoins du passé. » (Saint-Guénolé, 12 juillet 1920.)

Nous reconnaissons également cette nécropole de l'âge du Fer dans la description que fait Th. Monod de l'une des sépultures : «Je vous ai dit en un mot que l'autre jour, j'avais eu l'honneur de trouver une inhumation gauloise. Profondeur $=0,65$, squelette $=1,75$. Orientation pieds $N 20^{\circ}$ E. Pas de mobilier funéraire. L'avant-bras droit ramené à angle droit sur le corps, les jambes croisées (gauche dessus)» (8 septembre 1920). Ce dernier détail dans la description anthropologique nous permet d'identifier l'une des inhumations de Roz an Trémen où cette pratique (inhumation jambes croisées) a été observée à plusieurs reprises (Bénard et al., 1921).

À Roz an Trémen, Th. Monod réalisera le plan de localisation et le relevé d'ensemble de la nécropole. 


\section{... À Saint-Urnel...}

Cette même année 1920, au mois de septembre, Th. Monod participe à une autre fouille de nécropole, toujours sous la direction de $\mathrm{Ch}$. Bénard. Bien que la note soit rédigée en « nord Bretagne », il s'agit très vraisemblablement des toutes premières investigations sur la nécropole de Saint-Urnel à Plomeur (fig. 7 et fig. 8). Nous argumenterons ce point à la suite de la note de Th. Monod: "Cet après-midi, par un glorieux soleil de septembre, dans une atmosphère pure et transparente, par une douce lumière bien différente de la brutalité des rayons caniculaires, j'ai fait avec Besnard une tournée archéologique. Nous sommes passés par une certaine carrière à squelettes qui m'intriguait fort. Au fond de la carrière, de tous côtés, des ossements humains en vrac. Je me suis mis avec ardeur à l'ouvrage en débutant par un bras, ce bras m'a mené à une omoplate puis à un crâne. Je me rappellerai toujours ce vénérable crâne de guerrier portant à sa partie supérieure la marque de sa bravoure et la cause de sa mort sous la forme d'un trou: probablement quelque flèche de silex sera venue envoyer ad patres le colosse préhistorique. Car c'était un vrai colosse. Avec Besnard, nous avons dégagé tout le squelette: fémurs énormes, vertèbres de taille remarquable. Le crâne me paraît curieux: pommettes saillantes, capacité crânienne énorme. Quelle émotion dans la découverte de cet humain étendu dans son lit de pierres depuis des milliers d'années! Il y a probablement eu d'effroyables combats pouvant seuls expliquer pareille accumulation de débris humains. L'absence totale de tout métal dans le gisement, la présence d'un silex taillé ferait remonter ces cadavres à un âge très reculé: début du Néolithique, Paléolithique peut-être. » (septembre 1920).

Figure 8 : Fouilles du Groupe finistérien d'études préhistoriques sur la nécropole de Saint-Urnel (Plomeur, Finistère), 1923-1924.

Figure 8 : Excavation of the Saint-Urnel cemetery (Plomeur, Finistère), by the Groupe finistérien d'études préhistoriques, 1923-1924.

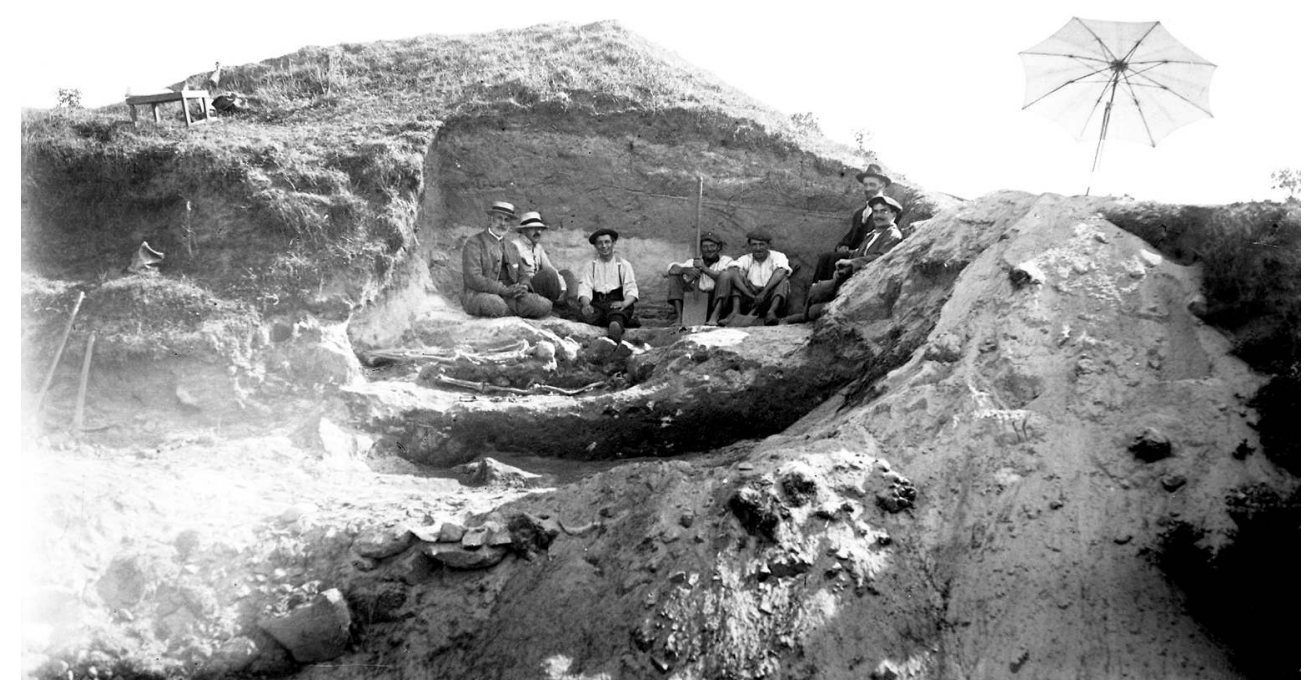

Au centre (béret noir) l'abbé Favret; à droite (barbe et chapeau noir) Charles Bénard. Center (black beret) the Abbet Favret, right (beard and black hat): Charles Bénard.

(c) Labo Archéosciences UMR 6566 CReAAH. 
Cette lettre à ses parents est agrémentée d'un croquis figurant le relevé stratigraphique de la fouille, avec un squelette, de la main de Th. Monod; mais cette découverte archéologique lui a également inspiré un poème composé de neuf quatrains, intitulé «Préhistoire » et illustré de sa main par des dessins de crânes et d'os stylisés. Ce poème est intégralement publié dans les Carnets (Monod, 1997, p. 24-25); nous en citons ici brièvement quelques vers :

«J'ai foulé de mon pas actuel et moderne

L'antique tumulus où le clan des chasseurs

Vint enterrer mon père, au temps des polisseurs

Et d'un bloc de granite obturer la caverne.

A genoux sur la glèbe au contact émouvant,

J'ai gratté de mes mains un sol pétri de crèmes... »

La fouille des sépultures enthousiasme le jeune homme: "Hier, grosse émotion: je déblayais une fouille sans grand espoir quand soudain à $60 \mathrm{~cm}$ de fond, je vois passer les osselets d'un tarse: alors, je travaille avec fureur pour mettre au jour un magnifique Gaulois : le crâne est tout à fait conservé.» (Saint-Guénolé, dimanche 5 septembre 1920.) Enthousiasme qu'il voudrait faire partager à ses parents : "Je voudrais vous montrer au fond de la fosse fraîchement ouverte, le squelette de quelque Gaulois ou le vase à incinération contenant les restes de quelque ancêtre plus vieux encore. » (Mercredi 8 septembre 1920.)

Il est possible, bien que peu probable comme nous le verrons plus loin, que Th. Monod ait participé aux campagnes de fouilles suivantes sur cette nécropole qui est alors considérée comme préhistorique. En effet, Ch. Bénard précise : «Pourtant, l'inhumation ne disparut jamais totalement et je puis en donner comme preuve le cas exceptionnel de deux magnifiques sépultures et celui d'une nécropole à étages d'inhumation que j'ai étudiées en SaintUrnel en Plomeur en 1922 avec M. Monod, docteur ès Sciences, attaché au Muséum d'histoire naturelle de Paris. » (Bénard, 1929, p. 220 ; fig. 7.) On peut se demander si le texte de Bénard ne confond pas les campagnes de fouilles de 1920 et 1922, dans la mesure où, de son côté, Th. Monod ne fera aucune mention de la campagne de 1922.

\section{...En passant par les Glénan}

43 À la fin du mois de juillet et au début du mois d'août 1920, Th. Monod s'occupe de prélever du "plankton » [sic] pour les études qu'il mène dans le cadre de la campagne océanographique autour de l'archipel des Glénan (Fouesnant, Finistère) ; il évoque aussi le patrimoine archéologique de l'archipel : "Il y a aussi, aux Glénans [sic] des mégalithes, des tumuli, etc. » (lettre postdatée du 8 août 1920, mais qui doit remonter plutôt au 20 ou 21 juillet). Puis, ailleurs : "Nous avions avec nous aux Glénans [sic] un abbé qui venait de fouiller un tumulus» (lettre du 25 juillet 1920). Il pourrait s'agir vraisemblablement de l'abbé Favret, si l'on se réfère à un courrier que Th. Monod adressait à P.-R. Giot le 13 juin 1953 : "C'était en 1920. Favret avait fouillé un tumulus aux Glénan et je me souviens que l'on se demandait s'il s'agissait d'une survivance relativement tardive (pour la région) ou d'une sépulture étrangère (Normands?).»

Au cours de cet été 1920, Ch. Bénard organise une campagne de fouille sur l'île du Loc'h dans l'archipel des Glénan, explorant une nécropole regroupant «des centaines" de coffres en pierre de deux types architecturaux différents renfermant, les uns des squelettes en position accroupie, et les autres des restes calcinés de crémations (cendres, charbons et os). Le commandant du Mistral, le lieutenant de vaisseau Masse, 
participe à ces travaux avec la réalisation d'un relevé schématique du site (Giot, 1996 : 37 ; Briard et al., 1989). Th. Monod participe également à cette expédition (Bénard et al., 1921) : «Ce matin, fouilles mégalithiques : j'ai trouvé un magnifique fragment de hache polie et un petit polissoir. Bénard a trouvé un très beau percuteur [...] Au revoir mes bien-aimés, je pars pour éventrer un tumulus près d'ici. Votre fils aimant » (25 juillet 1920).

Cependant, les recherches archéologiques dans l'archipel ne suscitent qu'assez peu de commentaires de la part de Th. Monod : «Le tumulus que nous avons fouillé aux Glénan doit être de la même époque normande [que celui de Groix, cf. Du Chatellier et Le Pontois, 1908], à en juger par les poteries de fond ; il n'y a que peu de siècles qu'on a abandonné la sépulture par tumulus dans les pays nordiques " (vendredi matin, [s. d., probablement en septembre], à bord du Mistral).

Cette mission de recherches préhistoriques aux Glénan vaudra à l'équipe, quelques années plus tard, des critiques très acerbes de la part du couple Marthe et Saint-Just Péquart (1927): reprenant leur inventaire des monuments (Bénard et al., 1921, p. 36-37); ils en critiquent pratiquement toutes les notices en dénonçant des erreurs d'interprétation : "Le menhir de l'île aux Moutons n'est qu'une saillie du sous-sol ; le tumulus de l'île Guiriden n'est qu'un amas de galets naturels; l'alignement de quartz de l'île Guiautec serait aussi un filon naturel, etc. " Quant aux coffres de l'île du Loc'h, fouillés par Bénard et ses confrères «les dégradations et les bouleversements occasionnés par les précédents fouilleurs ne peuvent que rendre désormais les observations bien difficiles. » (Péquart, 1927). Mais on doit reconnaître que l'abbé Favret et Ch. Bénard eux même faisaient, dès 1922, " amende honorable » en reconnaissant que les fouilles devaient "éviter les destructions aussi regrettables que celles des périodes antérieures " et s'engageaient vers une méthode impeccable d'observation scientifique, où le «bibelot d'étagère sera négligé comparativement aux documents d'ordres généraux et aux éléments de stratigraphie et de géologie non observés jusqu'ici. » (Favret et al., 1923).

Si l'archéologie des Glénan a suscité relativement peu de commentaires à Th. Monod, la découverte de l'archipel éveillera sa sensibilité à un paysage très « exotique » dans ses transparences marines. Dès 1920, il se réjouit : «Je me porte à merveille et cela ne sent pas le renfermé aux Glénans [sic] !» (25 juillet 1920). Ce sentiment de bien-être le suivra l'année suivante: «Cet archipel est unique avec son semis d'îlots granulitiques couronnés de cormorans. Quelques îles plus étendues renferment des landes, voire un étang aux bords envahis de plantes aquatiques. La pureté de l'eau est très remarquable et la même eau transparente vient baigner la grève de sable, la plage de galets ou le rocher abrupt tapissé de laminaires et de fucus. On se sent loin du monde dans ces coins absolument déserts et sauvages, aussi passionnants et attachants pour l'artiste que pour le savant. L'impression de calme et de paix est profonde, même angoissante : on perçoit avec acuité - par-delà l'émotion purement esthétique - le sentiment d'un salut cosmique nécessaire. Cette nature tout entière, cette terre, qui déroule sans trêve l'ellipse de son orbite, doivent être sauvées. ( (îles de Glénan, 6 septembre 1921.)

\section{Une page se tourne dans la vie de Th. Monod}

Une seule publication en co-signature marquera cette collaboration avec les archéologues bretons (Bénard et al., 1921; Hureau et Escuider, 2005), comportant une carte, deux plans et quatre dessins de crâne dus à Th. Monod, qui lui donne « pas mal de travail ». Et c'est toujours avec enthousiasme qu'il écrit : « Nous préparons sur nos fouilles 
un superbe mémoire avec cartes, plans, dessins, photographies, qui sera pas "piqué des hannetons". Vous voyez que je me lance dans l'édition. » (10 septembre 1920).

La campagne se termine pour lui sur un regret, celui de ne pouvoir emporter quelques morceaux des squelettes de Saint-Urnel avec qui il partage les étagères de son laboratoire de Penmarc'h, décision qu'il réprouve et commente ainsi : "Cette affaire de musée est bien embêtante: voilà deux squelettes entiers que je déterre et je n'en aurais pas un coccyx!» (29 septembre 1920). C'est manifestement le projet de futur musée préhistorique finistérien, en discussion entre Bénard et ses acolytes, qui est ici la cause de déception!

La mission se termine et laisse cependant Th. Monod en bons termes avec ses confrères et en particulier Ch. Bénard : "Chers, je pars demain pour Lutèce [...]. Bénard m'a dit toute l'estime qu'il avait pour moi, pour mon caractère, mon enthousiasme scientifique et ma moralité." (dimanche soir, 3 octobre 1920). Le journal tenu par W. et D. Monod, les parents de Théodore, rapporte un témoignage concordant: le Cdt Bénard leur rend visite et leur déclare que "Théodore est une des espérances de la science française ». Il rend hommage à l'attitude morale et au caractère du jeune homme, qu'on appelait en Bretagne "le petit savant» et qui, malgré son jeune âge, savait faire respecter ses convictions (Vray, 1994). Les événements des décennies suivantes démontreront toute la clairvoyance du jugement de Ch. Bénard!

51 En 1921, Th. Monod achève sa licence de sciences naturelles en passant son certificat de géologie et obtient une bourse du Muséum national d'histoire naturelle pour y effectuer sa thèse. Cette même année, l'office scientifique et technique des pêches maritimes organise une nouvelle campagne et offre à Th. Monod de participer à une nouvelle campagne océanographique de deux mois, cette fois à bord du garde-côte Pétrel qui le conduisit jusqu'à l'Aber Wrac'h, sans retour à Penmarc'h (Giot, 1996, p. 37). Ni ses carnets intimes de la période intermédiaire, ni ses lettres à ses parents, écrites depuis le bord, entre le 10 août et le 17 septembre, ne mentionneront d'expédition archéologique. De son propre aveu, les résultats scientifiques de la mission seront décevants, et même « maigrissimes ».

52 Les notes manuscrites de Th. Monod nous révèlent que, pendant cette campagne océanographique de deux mois (août et septembre 1921) qui le conduira à Concarneau, Lorient, Carantec..., il consacre son temps libre à l'apprentissage de l'hébreu, ce qui peut expliquer qu'il n'ait plus ni le temps, ni l'occasion de pratiquer l'archéologie.

En 1922, Th. Monod, nommé depuis janvier comme assistant au Muséum national d'histoire naturelle, au département "des pêches et productions coloniales d'origine animale ", est convié à participer à une nouvelle campagne océanographique à bord de l'Andrea III, un chalutier armé par la Marine nationale qui doit croiser le long des côtes bretonnes; il y restera embarqué du 6 au 30 août (Berne et Monod, 2010). D'un ton beaucoup plus solennel, ses lettres trahissent ce qu'il qualifie lui-même de "crise spirituelle violente » (carnet intime, année 1922) ; une déception amoureuse survenue au début de l'été (Berne et Monod, 2010) est probablement en partie responsable d'un regain de spiritualité, les manuscrits de Th. Monod n'étant désormais constitués que de discours théologiques fleuves : "J'ai connu, après les larmes qui soulagent, le froid désespoir qui ronge et qui glace. » (Feuillet du carnet intime, 1922).

Pourtant, au cours de ces années 1921-1922 et des suivantes, Ch. Bénard poursuit ses campagnes d'exploration archéologique, dans la région de La Torche, s'adjoignant 
d'ailleurs, entre autres, la collaboration de G. Monot - homonyme du précédent et commerçant à Pont-l'Abbé. Mais les "carnets » de Th. Monod ne comportent plus de mentions de découvertes archéologiques ni même de visites de sites, comme si, une fois émoussé, l'enthousiasme des premières expériences, le jeune chercheur avait déjà pris ses distances avec l'archéologie métropolitaine en rêvant déjà à d'autres horizons.

On ne sait rien de la suite des relations entre les deux hommes. Lorsqu'en 1930-1931, Ch. Bénard dirige l'expédition scientifique des "Cargos du désert» au Sahara, Th. Monod ne semble participer en rien à cette aventure, alors qu'il était déjà un saharien connu (Giot, 1996).

Peut-être parce que cette période de sa vie lui a laissé le goût doux-amer d'un premier chagrin d'amour, Th. Monod restera par la suite très discret sur cette expérience de jeunesse de la pratique de l'archéologie. Mais, à n'en pas douter, elle a contribué à sa formation intellectuelle et scientifique; son attrait pour la préhistoire africaine n'apparaît alors plus comme le fruit du hasard : "J'ai participé en 1928 à la découverte du squelette fossile de l'homme d'Asselar (Sahara soudanais). Les récoltes d'industries anciennes ont été très abondantes au cours de mes voyages sahariens à l'occasion desquels j'ai découvert le célèbre gisement de bifaces d'El-Beyyed, ainsi que plusieurs sites importants de galets aménagés. » (Vray, 1994, p. 387).

Les auteurs remercient chaleureusement $\mathrm{M}^{\text {me }}$ Alice Lemaire et ses collègues du service $\mathrm{du}$ patrimoine (direction des bibliothèques et de la documentation) du Muséum d'histoire naturelle de Paris pour leur accueil et leur aide dans la consultation du fonds d'archives de Théodore Monod, ainsi que Monsieur Ambroise Monod qui a chaleureusement accueilli ce projet et nous a aimablement autorisés à utiliser et à reproduire le portrait de Th. Monod.

\section{BIBLIOGRAPHIE}

MONOD Th., 1920 - Mission sur le Mistral (juillet-septembre 1920) : correspondance active à ses parents. Missions océanographiques, Carnets de Route, archives personnelles de Th. Monod, Muséum national d'histoire naturelle, cote Ms MDP, 8.4, Lettres illustrées.

MONOD Th., 1921 - Mission sur le Pétrel (août-septembre 1921) : correspondance active à ses parents. Missions océanographiques, Carnets de route, archives personnelles de Th. Monod, Muséum national d'histoire naturelle, cote Ms MDP, 8.5, Lettres illustrées.

MONOD Th., 1922 - Mission sur le l'Andréa III (août 1922) : correspondance active à ses parents. Missions océanographiques, Carnets de Route, archives personnelles de Th. Monod, Muséum national d'histoire naturelle, cote Ms MDP, 8.6, Lettres illustrées.

MONOD W. et D., 1902-1930 - Journal de l'enfance de Théodore Monod rédigé par ses parents, 1902-1930, archives personnelles de Th. Monod, Muséum national d'histoire naturelle, Cote Ms MDP 1.1, 1 carnet. 
BÉNARD LE PONTOIS Ch., 1929 - Le Finistère préhistorique, Publications de l'Institut international d'anthropologie, $\mathrm{n}^{\circ}$ 3, Librairie E. Noury, Paris.

BÉNARD Ch., FAVRET (abbé) et BOISSELIER G., 1920 - « Importance archéologique de la région de la presqu'île de La Torche », Bulletin de la Société archéologique du Finistère, XLVII, p. 172-192.

BÉNARD Ch., FAVRET (abbé), BOISSELIER G. et MONOD Th., 1921 - « Deuxième campagne de fouilles dans la région de La Torche et les îles Glénan », Bulletin de la Société archéologique du Finistère, XLVIII, p. 22-44.

BÉNARD ch., FAVRET (abbé), BOISSELIER G. et MONOT G.A., 1922 - « Troisième campagne de fouilles en pays bigouden », Bulletin de la Société archéologique du Finistère, XLIX, p. 37-50.

BERNE M. et MONOD A. (dir.), 2010 - Théodore Monod. Archives d'une vie, Coédition Le Chêne, Muséum d'histoire naturelle, Paris.

BRIARD J, GUEGUEN M. et LE ROUX C.-T., 1989 - « L'âge du Bronze aux îles Glénan, Fouesnant, Finistère », Bulletin de l'AMARAI, 2, p. 47-54.

CHAIGNEAU C., CASSEN S. et LE GAUDION Y., 2009 - « Bibliographie générale sur Locmariaquer », in CASSEN S. (dir.), Autour de la Table. Explorations archéologiques et discours savants sur des architectures néolithiques à Locmariaquer, Morbihan (Table des Marchands et Grand Menhir), Laboratoire de recherches archéologiques (LARA), CNRS (UMR 6566), université de Nantes, p. 418-457.

COLLECTIF, 2010 - La vie de Théodore Monod racontée par ses proches à partir d'archives, Coédition Le Chêne, Muséum d'histoire naturelle, Paris.

CANS R., 2002 - Théodore Monod, savant tous terrains. Biographie, Sang de la Terre, Paris.

DAIRE M.-Y., 2005 - Les Stèles de l'âge du Fer dans l'Ouest de la Gaule. Réflexions sur le monde des morts et le monde des vivants, Les dossiers du CeRAA, $\mathrm{n}^{\circ} \mathrm{AB}$, Rennes.

DAIRE M.-Y. et LÓPEZ-ROMERO E., 2011 - Au fil de l'eau..., Catalogue d'exposition, éd. UMR CReAAH, université de Rennes 1, Rennes.

DESAILLY M., 1926 - « Séance du 23 décembre 1926 », Bulletin de la Société préhistorique française, 23, p. 300 .

DU CHATELLIER P. et LE PONTOIS L., 1908 - « La sépulture scandinave à barque de l'île de Groix », Bulletin de la Société archéologique du Finistère, XXXV, p. 3-98.

FAVRET A., BÉNARD ch. et MONOT G., 1923 - «Quatrième campagne de fouilles préhistoriques dans le Finistère ", Bulletin de la Société archéologique du Finistère, L, p. 83-93.

GIOT P.-R., 1996 - « Théodore Monod en Finistère », Bulletin de la Société archéologique du Finistère, CXXV, p. 36-38.

HUREAU J.-C. et ESCUDIER D., 2005 - Théodore Monod : 90 ans de publications, Publications scientifiques du Muséum d'histoire naturelle, Paris.

JARRY I., 2001 - Théodore Monod, Petite bibliothèque Payot (169), Paris.

LECA M. et JACQUARD A., 1997 - Le chercheur d'absolu. Théodore Monod, Le Cherche Midi, Paris.

LÓPEZ-ROMERO E. et DAIRE M.-Y., 2013 - « The ICARE project: an insight into the formation and consolidation of archaeology in western France (ca. 1850-1990) », Bulletin for History of archaeology, 23(1):3, p. 1-11 DOI : [https://dx.doi.org/10.5334/bha.2313]. 
MARATRAY R.D. (de), 1920a - « La nouvelle mission océanographique », La France de l'Ouest, 2 juillet 1920, « informations », Archives du Cdt Bénard, archives du laboratoire archéosciences, UMR 6566, CReAAH, Rennes.

MARATRAY R.D. (de), 1920b - « La campagne océanographique », La France de l'Ouest, 27 octobre 1920, Archives du Cdt Bénard, archives du laboratoire archéosciences, UMR 6566, CReAAH, Rennes. MONOD Th., 1921 - Influence de la température sur la composition qualitative du plankton. Contribution à l'étude de la faune pélagique d'été du sud-ouest de la Bretagne. Mémoire composé à la suite des campagnes océanographiques sur le Mistral (1920) et le Pétrel (1921), Manuscrit Cote MS Md 16 (dossier 4), Archives du MNHN.

MONOD Th., 1922 - «Influence de la température sur la composition qualitative du plankton ", Revue générale des sciences pures et appliquées, 33-3, p. 69.

MONOD Th., 1926 - $1^{\text {re }}$ thèse : Les Gnathiidae, essai monographique. $2^{\mathrm{e}}$ thèse : La région de la Basse-Seulles : étude bionomique. Thèse du Muséum national d'histoire naturelle, Presses universitaires de France, Paris.

MONOD C., 1997 - Les carnets de Théodore Monod. Dans l'intimité du « fou du désert », Le Pré aux Clercs.

PÉQUART M. et PÉQUART S.-J., 1927 - « Dolmen de Brunec (îles Glénan) », Bulletin de la Société

archéologique du Finistère, LIV, p. 73-83.

PEYRON J.-M. et ABGRALL Ch., 1904 - Diocèse de Quimper et de Léon. Notices sur les paroisses, Quimper.

RÉVEILLÈRE J. (de La), 1903 - « Légendes et superstitions préhistoriques. Danses autour d'un menhir », Revue des traditions populaires, CXXXVII, p. 253.

VRAY N., 1994 - Monsieur Monod. Scientifique, voyageur et protestant. Biographie, Actes Sud, Paris.

\section{RÉSUMÉS}

La vie et l'œuvre scientifique de Théodore Monod, savant et naturaliste français, ont marqué le $\mathrm{xx}^{\mathrm{e}}$ siècle. Mais ici, c'est un épisode beaucoup moins connu de sa vie que les auteurs ont retracé à partir d'archives inédites et privées pour la plupart: en effet, pendant ses années de jeunesse, Th. Monod a non seulement côtoyé les pionniers de l'archéologie bretonne mais il a aussi participé à plusieurs campagnes de prospections, relevés et fouilles archéologiques dans le Finistère. Cette note apporte ainsi une contribution originale à l'historiographie de la préhistoire bretonne.

The life and scientific work of Theodore Monod, French naturalist and scientist, marked the 20th century. But here, a much less known episode of his life has been traced from mainly unpublished and private archives. During his early years, Th. Monod was not only acquainted with the pioneers of Breton archeology but he also participated in several fieldwork campaigns, surveys and excavations in the Finistère. This note therefore provides an original contribution to the historiography of the Prehistory of Brittany.

El trabajo y la vida científica de Theodore Monod, científico y naturalista francés, marcaron el siglo XX. Pero aquí, es un episodio mucho menos conocido en su vida que los autores han rastreado a partir de archivos inéditos y privados para la mayoría de ellos: de hecho, durante sus primeros años, Monod no sólo ha conocido a los pioneros de la arqueología de Bretaña, pero 
también ha participado en varias campañas de encuestas, estudios y excavaciones en Finistère. Este artículo lleva así una contribución original a la historiografía de la prehistoria de Bretaña.

\section{INDEX}

Keywords : Théodore Monod, prehistory, Metal Ages, Brittany, Finistère, Charles Bénard, historiography

Palabras claves : Theodore Monod, prehistoria, Bretaña, Finistère, Charles Bénard, historiografía

Mots-clés : Théodore Monod, préhistoire, protohistoire, Bretagne, Finistère, Charles Bénard, historiographie

\section{AUTEURS}

\section{MARIE-YVANE DAIRE}

UMR 6566 CReAAH « Centre de recherche en archéologie, archéosciences, histoire », Laboratoire archéosciences, Rennes, France. (marie-yvane.daire@univ-rennes1.fr)

\section{ELÍAS LÓPEZ-ROMERO}

Durham University, Department of Archaeology. (elias.lopez-romero@durham.ac.uk)

\section{CATHERINE LE GALL}

UMR 6566 CReAAH « Centre de recherche en archéologie, archéosciences, histoire », Laboratoire archéosciences, Rennes, France. (catherine.le-gall@univ-rennes1.fr) 